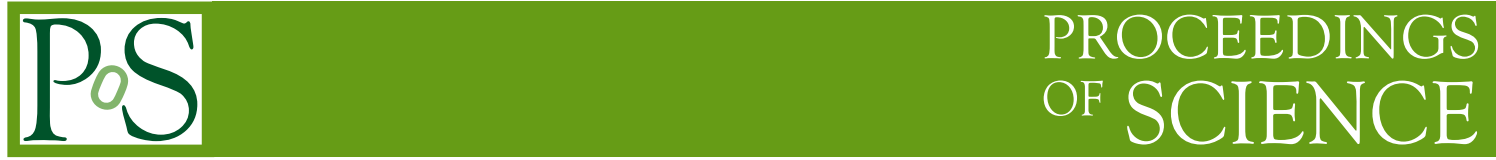

\title{
The B Meson Decay Constant in Full QCD
}

\author{
Junko Shigemitsu ${ }^{* a}$, Alan Gray $^{a}$, Matthew Wingate ${ }^{b}$, Christine Davies $^{c}$, Emel \\ Gulez $^{a}$, Peter Lepage ${ }^{d}$, Quentin Mason ${ }^{e}$, Matthew Nobes ${ }^{d}$ \\ ${ }^{a}$ Physics Department, The Ohio State University, Columbus, OH 43210, USA. \\ ${ }^{b}$ Institute for Nuclear Theory, University of Washington, Seattle, WA 98195, USA. \\ ${ }^{c}$ Department of Physics and Astronomy, University of Glasgow, Glasgow, G12 8QQ, UK \\ ${ }^{d}$ LEPP, Cornell University, Ithaca, NY 14853, USA. \\ ${ }^{e}$ DAMTP, University of Cambridge, Cambridge, UK.
}

\begin{abstract}
We calculate the $B$ meson decay constant $f_{B}$ and the ratio $f_{B_{S}} / f_{B}$ in full QCD using the MILC collaboration unquenched gauge configurations. We employ a nonrelativistic QCD action for the heavy $b$ quark and the highly improved (AsqTad) staggered quark action for the light sea and valence quarks. The good chiral properties of the latter action allow for an accurate chiral extrapolation to physical up and down quarks.
\end{abstract}

XXIIIrd International Symposium on Lattice Field Theory

25-30 July 2005

Trinity College, Dublin, Ireland

\footnotetext{
${ }^{*}$ Speaker.
} 


\section{Introduction}

The phenomenon of neutral $B_{d}-\bar{B}_{d}$ mixing is now well established experimentally and the mass difference $\Delta M_{d}$ is known with an accuracy of about $\sim 1 \%$. The goal is to extract the CKM matrix element $V_{t d}$ using the Standard Model relation,

$$
\Delta M_{d} \propto\left|V_{t d} V_{t b}\right|^{2}\left(f_{B}^{2} \hat{B}_{B}\right)
$$

Here $f_{B}$ is the $B$ meson decay constant and $B_{B}$ its so-called bag parameter, and the current bottle neck in the $V_{t d}$ program comes from the large theoretical uncertainty in the combination $f_{B} \sqrt{B_{B}}$. Lattice QCD provides a way to determine $f_{B}$ and $f_{B} \sqrt{B_{B}}$ from first principles QCD calculations. We report here on recent determinations of $f_{B}$ and of the ratio $f_{B_{s}} / f_{B}$ by the HPQCD collaboration [1]. Several errors that have plagued previous lattice calculations of these quantities, in particular those coming from quenching (or partial quenching) and from chiral extrapolations, have either been removed or significantly reduced. We achieve this by working with the MILC collaboration $N_{f}=2+1$ dynamical configurations [2] and by employing the highly improved (AsqTad) staggered quark action [3] for both the sea quarks and for the light valence quarks inside heavy-light mesons [4]. Simulations are carried out with light quark masses between $m_{s}$, the strange quark mass, and $m_{s} / 8$. Our masses are light enough to be in a regime where chiral perturbation theory is valid and as a consequence extrapolations to physical up and down quarks can be carried out accurately. For the heavy $b$ quark we employ the same nonrelativistic QCD action used in a recent study of the $\Upsilon$ system [5]. Our main results are given by,

$$
f_{B_{s}} / f_{B}=1.20(3)(1)
$$

and

$$
f_{B}=216(9)(19)(4)(6) \mathrm{MeV} \text {. }
$$

The first error in eq.(1.2) comes from statistics plus chiral extrapolations and the second is an estimate of residual errors (not cancelled by taking a ratio) due to discretization, relativistic and operator matching effects. In eq.(1.3) the errors are, from left to right, due to statistics plus chiral extrapolations plus lattice spacing uncertainties, higher order operator matching, discretization and relativistic corrections plus $b$ quark mass tuning, respectively.

\section{Simulation Details}

Table I summarizes the lattices that were used in our simulations. Most of our results come from the four "coarse" MILC ensembles with lattice spacing $a$ around $0.12 \mathrm{fm}$, but we also accumulated data on two "fine" ensembles with lattice spacing around $0.087 \mathrm{fm}$.

The relevant hadronic matrix element is that of the heavy-light axial vector current $A_{\mu}$ between the $B$ meson state and the hadronic vacuum. In the $B$ rest frame one has,

$$
\left\langle 0\left|A_{0}\right| B\right\rangle=M_{B} f_{B} .
$$




\begin{tabular}{|c|c|c|c|c|c|}
\hline$u_{0} a m_{f}$ & $a^{-1}(\mathrm{GeV})$ & $n_{\text {conf }}$ & $n_{s r c}$ & $u_{0} a m_{q}$ & $m_{q} / m_{s}$ \\
\hline \hline Coarse & & & & & \\
$0.005^{*}$ & $1.623(32)^{\dagger}$ & 399 & 4 & 0.005 & 0.125 \\
& & & 4 & 0.040 & 1.000 \\
$0.007^{*}$ & $1.622(32)^{\dagger}$ & 397 & 4 & 0.007 & 0.175 \\
& & & 4 & 0.040 & 1.000 \\
0.010 & $1.596(30)$ & 568 & 4 & 0.005 & 0.125 \\
& & & 2 & 0.010 & 0.250 \\
& & & 2 & 0.020 & 0.500 \\
& & & 1 & 0.040 & 1.000 \\
0.020 & $1.605(29)$ & 486 & 2 & 0.020 & 0.500 \\
& & & 1 & 0.040 & 1.000 \\
\hline Fine & & & & & \\
$0.0062^{*}$ & $2.258(32)$ & 465 & 4 & 0.0062 & 0.200 \\
& & 472 & 4 & 0.031 & 1.000 \\
$0.0124^{*}$ & $2.312(31)$ & 496 & 4 & 0.0124 & 0.400 \\
& & & 4 & 0.031 & 1.000 \\
\hline
\end{tabular}

Table 1: Simulation Details. $m_{f}\left(m_{q}\right)$ denotes sea (valence) quark masses. A † means the lattice spacing was determined through $r_{1}$. For all other ensembles the $\Upsilon 2 \mathrm{~S}-1 \mathrm{~S}$ splitting was used. A * denotes ensembles that are new since LAT'04. $n_{s r c}$ is the number of light quark source points. $u_{0}=[\text { plaq }]^{1 / 4}$ is the link variable the MILC collaboration uses in their normalisation of quark masses.

Through $\mathscr{O}\left(\alpha_{s} / M\right)$, this matrix element can be written in terms of three lattice currents [6],

$$
\begin{aligned}
& J_{0}^{(0)}(x)=\bar{q}(x) \Gamma_{0} Q(x), \\
& J_{0}^{(1)}(x)=\frac{-1}{2 M_{0}} \bar{q}(x) \Gamma_{0} \gamma \cdot \nabla Q(x), \\
& J_{0}^{(2)}(x)=\frac{-1}{2 M_{0}} \bar{q}(x) \gamma \cdot \overleftarrow{\nabla} \gamma_{0} \Gamma_{0} Q(x),
\end{aligned}
$$

and one has

$$
\begin{aligned}
\left\langle A_{0}\right\rangle= & \left(1+\alpha_{s} \tilde{\rho}_{0}\right)\left\langle J_{0}^{(0)}\right\rangle+ \\
& \left(1+\alpha_{s} \rho_{1}\right)\left\langle J_{0}^{(1), s u b}\right\rangle+\alpha_{s} \rho_{2}\left\langle J_{0}^{(2), s u b}\right\rangle \\
J^{(i), s u b}= & J^{(i)}-\alpha_{s} \zeta_{10} J^{(0)} .
\end{aligned}
$$

This expression is correct through $\mathscr{O}\left(\frac{\Lambda_{Q C D}}{M}, \alpha_{s}, a \alpha_{s}, \alpha_{s} \frac{\Lambda_{Q C D}}{M}, \alpha_{s} \frac{1}{a M}\right)$ The one-loop matching coefficients, $\rho_{i}$ and $\zeta_{10}$, have been calculated in [7]

\section{The Ratio $f_{B_{s}} / f_{B}$}

In Fig.1 we show results for the ratio $\xi_{\Phi} \equiv \Phi_{s} / \Phi_{q}$, where $\Phi_{q} \equiv f_{B_{q}} \sqrt{M_{B_{q}}}$ and similarly for 
$q \rightarrow s$. The full curve is a fit to the staggered chiral perturbation theory $(S \chi P T)$ formulas of Aubin\&Bernard [8] which include contributions from $\mathscr{O}\left(a^{2}\right)$ lattice artifacts specific to the staggered quark action that we employ. We have also tried continuum $\chi P T$ formulas without the $\mathscr{O}\left(a^{2}\right)$ terms and simple linear chiral extrapolations with no chiral logarithms at all (see [1] for more details). All these different chiral extrapolations agree to within 3\%. Our data points lie sufficiently close to the chiral limit so that only a small and mild chiral extrapolation is required. Details of how the extrapolations are carried out become less important and chiral extrapolation uncertainties are significantly reduced. From the $S \chi P T$ curve of Fig. 1 we find at the physical point $m_{q} / m_{s}=1 / 27.4$, the value $\xi_{\Phi}^{(\text {phys. })}=1.21(3)$. This leads to our final result for $f_{B_{s}} / f_{B}=\xi_{\Phi}^{(\text {phys. })} \sqrt{\frac{M_{B}}{M_{B_{s}}}}$ given in eq.(1.2).

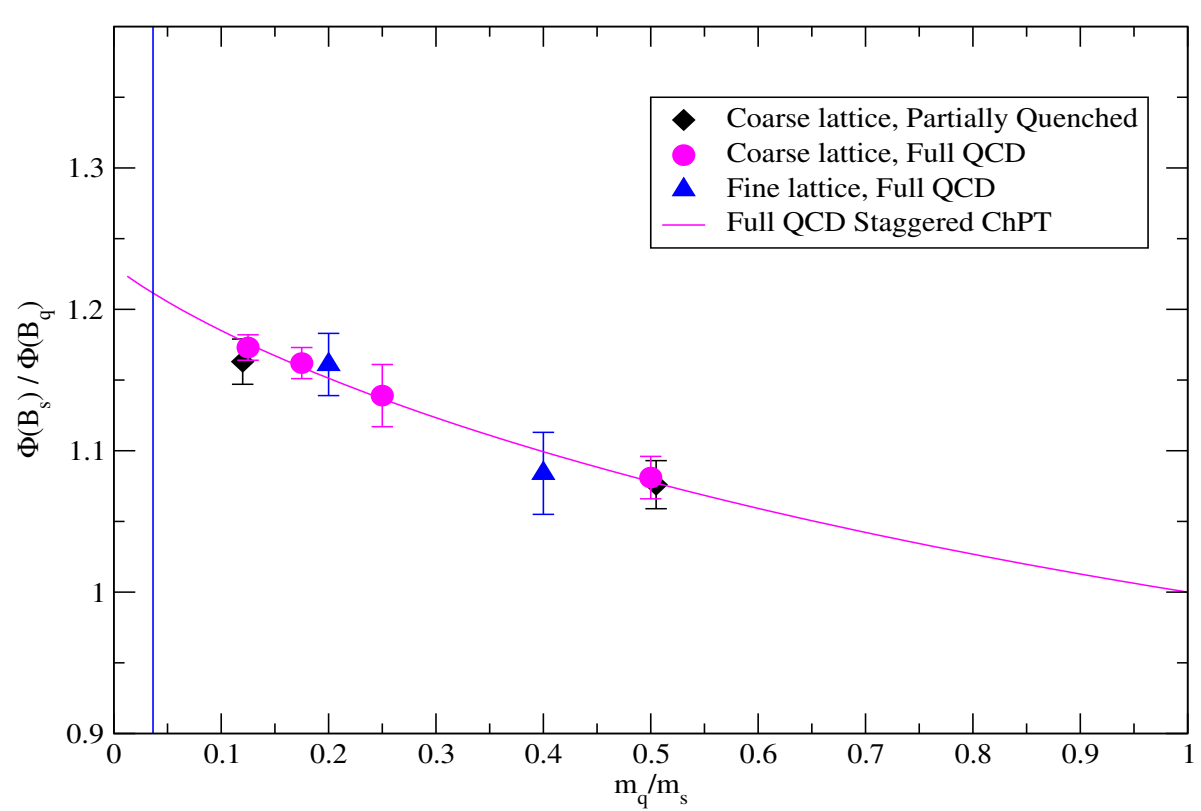

Figure 1: The ratio $\xi_{\Phi}=\Phi_{s} / \Phi_{q}$ versus $m_{q} / m_{s}$. The full line through the data shows a fit to full QCD staggered $\chi P T$ [8]. Only statistical errors are shown. The vertical line denotes the physical chiral limit.

\section{The Decay Constants $f_{B}$ and $f_{B_{s}}$}

Fig. 2 shows data for $\Phi_{q}$ together again with a $S \chi P T$ fit curve. The errors on the data points include both statistical errors and uncertainties in the scale $a^{-3 / 2}$ with the latter dominating in most cases. Different chiral extrapolation ansaetze lead to a spread of $\sim 4 \%$ which we take to be the combined statistics plus scale $\left(a^{-1}\right)$ plus chiral extrapolation uncertainty. Our final result for $f_{B} \equiv \Phi^{(\text {phys. })} / \sqrt{M_{B}}$ together with all the statistical and systematic errors is given in eq.(1.3). Table 2 lists the source and sizes (in percent) of the different errors.

Fig.3 shows the $B_{s}$ meson decay constant $f_{B_{s}}$ as a function of the sea quark mass. One sees that the sea quark mass dependence is very mild. Furthermore comparison of the coarse and fine 


\begin{tabular}{|c|c|}
\hline source of error & size of error $(\%)$ \\
\hline \hline $\begin{array}{c}\text { statistics }+ \text { scale }\left(a^{-1}\right) \\
+ \text { chiral extrapolation }\end{array}$ & $4 \%$ \\
\hline two-loop matching & $9 \%$ \\
\hline discretization & $2 \%$ \\
\hline relativistic $+b$ mass tuning & $3 \%$ \\
\hline \hline Total Error & $10 \%$ \\
\hline
\end{tabular}

Table 2: Error table for $f_{B}$

lattice results indicates that discretization errors are also small. In fig. 3 we also show our previous published result for $f_{B_{s}}$ based mainly on simulations at one lattice spacing and one sea quark mass [9]. Our published value is $f_{B_{s}}=260(29) \mathrm{MeV}$ with the error dominated by uncertainty in higher order matching of the lattice heavy-light current. Although we now have much more data for $f_{B_{s}}$ at several sea quark masses and at two lattice spacings (all consistent with the published value), we forego quoting an updated value at the present time. We opt to wait until two-loop operator matching becomes available.

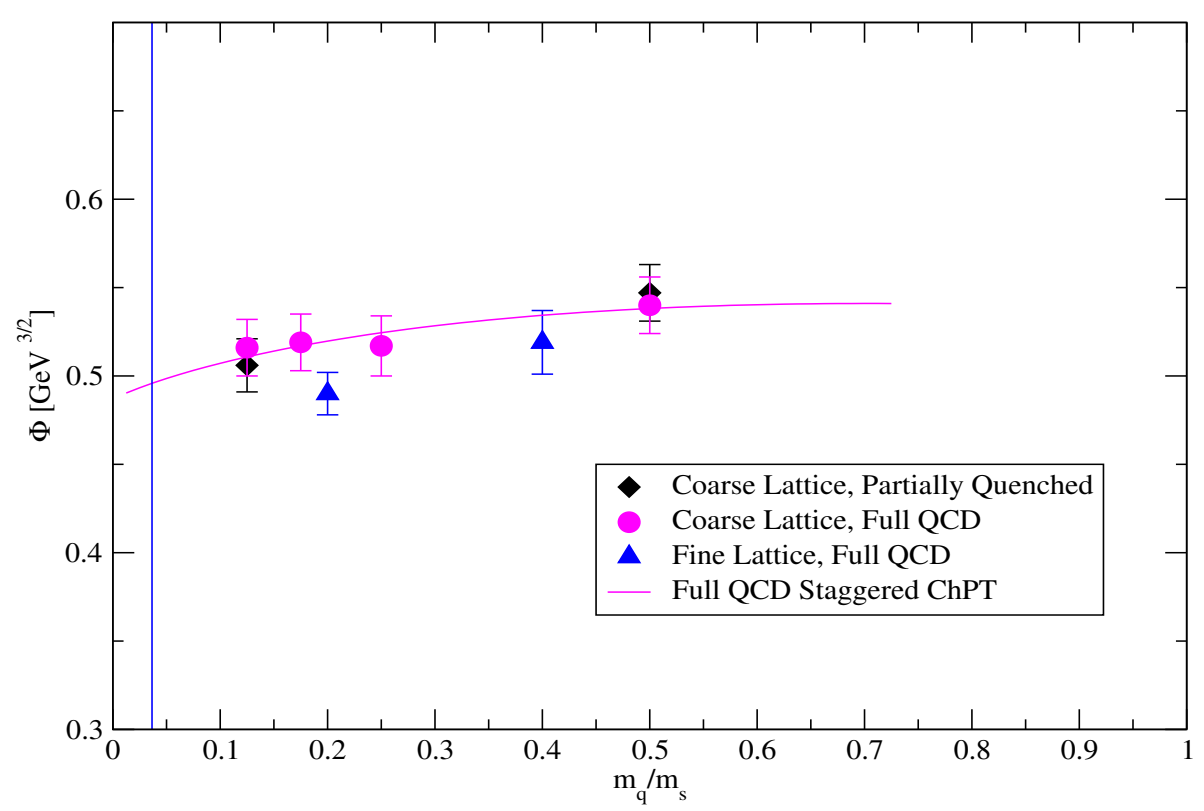

Figure 2: $\Phi_{q}$ versus $m_{q} / m_{s}$. Errors include statistical and $a^{-1}$ uncertainties.

\section{Summary}

There is urgent need for accurate theory input in Heavy Quark Physics today, in particular in 
connection with studies of $B$ meson decays and mixing phenomena. Considerable progress has been achieved recently by the lattice community but much work still remains. The main progress has come from realistic vacuum polarization in lattice simulations and better control over chiral extrapolations. In order to further reduce theory errors, higher order matching of lattice heavylight currents and four-fermion operators will be crucial.

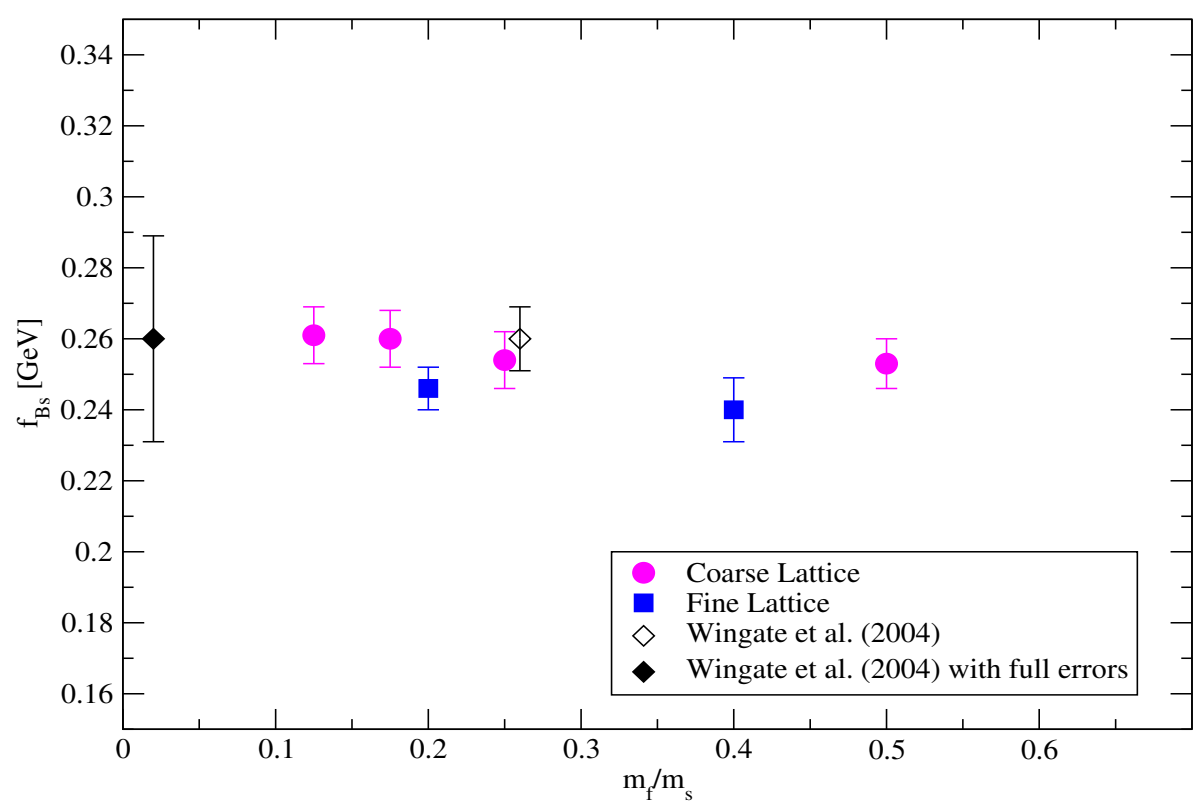

Figure 3: $f_{B_{S}}$ versus the sea quark mass. Errors include statistical and $a^{-1}$ uncertainties.

\section{References}

[1] A.Gray et al.; hep-lat/0507015.

[2] C.Bernard et al.; Phys. Rev. D64, 054506 (2001).

[3] S.Naik, Nucl. Phys. B316, 238 (1989); G.P.Lepage, Phys. Rev. D59, 074501 (1999); J.Lagae and D.Sinclair, ibid. 59, 014511 (1999); K.Originos et al., ibid. 60, 054503 (1999); C.Bernard et al., ibid. 61, 111502 (2000).

[4] M.Wingate et al.; Phys. Rev. D67, 054505 (2003).

[5] A.Gray et al.; hep-lat/0507013, to appear in Physical Review D.

[6] C.Morningstar and J.Shigemitsu; Phys. Rev. D57, 6741 (1998).

[7] E.Gulez et al.; Phys. Rev. D69; 074501 (2004).

[8] C.Aubin and C.Bernard; Nucl. Phys. B (Proc. Suppl.)140, 491 (2005);

W.Lee and S.Sharpe; Phys. Rev. D60, 114503 (1999);

C.Bernard, private communication.

[9] M.Wingate et al.; Phys. Rev. Lett. 92, 162001 (2004). 